\title{
ESTUDO DA EMISSÃO DOS GASES DE COMBUSTÃO PROVENIENTES DA QUEIMA DO DIESEL S10 E S50
}

\author{
L.A.D. KOSLOWSKI ${ }^{1}$, S.LICODIEDOFF ${ }^{1}$, T.M.N OLIVEIRA ${ }^{2}$, S.H.W. MEDEIROS ${ }^{2}$ \\ C. VAZ ${ }^{2}$, H.G. RIELLA ${ }^{1}$
}

${ }^{1}$ Universidade Federal de Santa Catarina, Departamento de Engenharia Química e Engenharia de Alimentos

${ }^{2}$ Universidade da Região de Joinville - Univillle, Departamento de Engenharia Química

E-mail para contato: lucianoandre@yahoo.com

\begin{abstract}
RESUMO - Pesquisadores tem desenvolvidos trabalhos de forma a avaliar e compreender o efeito da emissão de gases da combustão provenientes do transporte motorizado na atmosfera. $O$ processo de combustão, sendo uma oxidação a alta temperatura, gera óxido de nitrogênio $\left(\mathrm{NO}_{\mathrm{x}}\right)$, dióxido de carbono $\left(\mathrm{CO}_{2}\right)$, monóxido de carbono $(\mathrm{CO})$ e possivelmente poluentes perigosos como os Hidrocarbonetos Policíclicos Aromáticos (HPA's). O presente estudo teve por objetivo avaliar comparativamente a emissão de gases provenientes da queima do diesel S10 e diesel S50 em uma coluna de absorção de leito fixo acoplada em um motor de combustão estacionário. Os resultados mostraram para o diesel S10 concentrações médias de HPA de $36 \mu \mathrm{g} / \mathrm{L}, \mathrm{CO} 1660 \mathrm{mg} / \mathrm{N} . \mathrm{m}^{3}$; $\mathrm{NO}_{\mathrm{X}} 15 \mathrm{ppm}$; e para o diesel S50 concentrações de HPA $53 \mu \mathrm{g} / \mathrm{L}$ CO mg/N.m ${ }^{3}$; $\mathrm{NO}_{\mathrm{X}} 18 \mathrm{ppm}$.
\end{abstract}

\section{INTRODUÇÃO}

A crescente demanda energética causa pressões intensas para o uso das reservas mundiais de petróleo como fonte de combustível, promovendo o esgotamento desse recurso natural e a poluição do meio ambiente associada à sua queima, sendo que, na atual década, a maior parte da energia gerada e consumida no planeta é proveniente da queima de combustíveis fósseis, especificamente o petróleo, o carvão e o gás natural. Considerando esse aspecto, há um interesse renovado em utilizar fontes alternativas de combustíveis menos poluentes, visando a minimização da poluição e a redução do consumo de fontes não renováveis

O óleo diesel um derivado do petróleo utilizado amplamente no Brasil como combustível, é constituído principalmente por hidrocarbonetos de cadeia longa, por enxofre, nitrogênio e oxigênio, estes três últimos em baixas concentrações (Kozerski, 2006). O transporte de cargas no Brasil está apoiado, principalmente, no uso de veículos movidos por motores a diesel, por via rodoviária. Para atender o mercado nacional, as refinarias da Petrobras são operadas com prioridade para a produção desse combustível, o qual a produção corresponde em torno de 34\% do volume do petróleo processado no país (Ferrari et al., 2005).

Os poluentes emitidos pela combustão do diesel causam problemas ecológicos reconhecidos em todo o mundo, como a destruição da camada de ozônio, aumento do 


\section{9 a 22 de outubro de 2014 \\ Florianópolis/SC}

aquecimento global e chuva ácida (Knothe, 2005). Os motores a diesel de embarcações emitem material particulado, fumaça preta e carregam diversos compostos carcinogênicos de elevado impacto à saúde humana e aos ecossistemas. Algumas investigações para reduzir os poluentes dessas emissões são de fundamental importância para a saúde humana, meio ambiente e para a prosperidade da vida na Terra (Correa, 2006).

Os motores movidos a diesel são a maior fonte de poluição atmosférica do planeta. Esse combustível possui alto potencial para o aquecimento global da descarga como o $\mathrm{CO}_{2}$ pela queima em veículos automotores. Muitas doenças pulmonares estão ligadas ao material particulado emitido pelos veículos a diesel incluindo poeira, fuligem e fumaça. O biodiesel é considerado um combustível não tóxico e biodegradável (Tomaszewski, 2006). Comparado ao óleo diesel, o biodiesel não produz enxofre, emite menos dióxido de carbono, monóxido de carbono e mais oxigênio. A maior quantidade de oxigênio livre conduz à combustão completa e uma emissão reduzida. As emissões do biodiesel são muito menores em comparação com as emissões de diesel, sendo assim promissor o uso dessa alternativa para a promoção de um ambiente menos poluído (Schroeder, 2013).

A composição dos gases de exaustão da queima do diesel é muito complexa, sendo composta por três fases: sólidos, líquidos e gases. Quando os motores a diesel são operados em condições oxidantes, o que contribui para uma boa economia de combustível e resulta em menor produção de $\mathrm{CO}_{2}$, em um processo de combustão operando em temperaturas menores e com formação e consequente emissão minimizada das quantidades de $\mathrm{NOX}, \mathrm{CO}$ e hidrocarbonetos (Tsukue et al., 2010). O NOx participa em diversos fenômenos atmosféricos globais como por exemplo o Smog fotoquímico, a depleção do ozônio, chuva ácida doenças respiratórias graves como bronquite, tosse e edema pulmonar. Deste modo, este trabalho apresenta a partir da construção de uma coluna de absorção de leito fixo em aço inox, a quantificação dos gases da combustão do diesel S10 e S50 e de HPA's solubilizados em água.

\section{MATERIAIS E MÉTODOS}

\subsection{Materiais}

As amostras de diesel S10 e S50 utilizadas neste trabalho foram provenientes de um mesmo fornecedor da região de São Bento do Sul - SC. A diferença básica entre o diesel S10 e o diesel S50 é a quantidade de enxofre presente no diesel, que passou de 50 partes por milhão (S50) para 10 partes por milhão (S10), o que reduz a quantidade de NOx, SOx e outros materiais particulados provenientes da sua combustão. O diesel S-10 sofre um processo de refino diferente, o que eleva seu nível de cetano. O diesel S-50 apresenta índice 46 e o S10 tem índice de cetano de 48.

\subsection{Quantificação dos gases da combustão e HPA's}

Para realizar a combustão das amostras, foi utilizado um motor estacionário. A este motor estava ligado um sistema de exaustão acoplado a uma coluna de absorção de leito fixo, montada em aço inox, empregada para absorver os gases de combustão de diesel, apresentada na Figura 1. A leitura dos valores de NOx e CO foram realizadas na extremidade do tubo de descarga e a leitura dos HPA foi realizada nas amostras de água provenientes do processo de absorção. 
As colunas de absorção de leito fixo são os processos mais utilizados para a despoluição de gases, no qual a remoção química dos poluentes ocorre por absorção (Couvert, Sanchez et al., 2008). Muito embora a transferência de massa nesse processo seja muito mais intensa que aquela que ocorre em processos naturais, essa metodologia é necessária visando concentrar as emissões na água até o ponto de saturação e dessa forma podendo-se estabelecer diluições para os testes de toxicidade, bem como a quantificação do material solubilizado. A coluna foi montada em aço inox resistente à corrosão por água salgada, visando a não contaminação da água por outros materiais que não a fumaça emitida pelo escapamento do motor estacionário. Como recheio da coluna, foram utilizados fragmentos de porcelana.

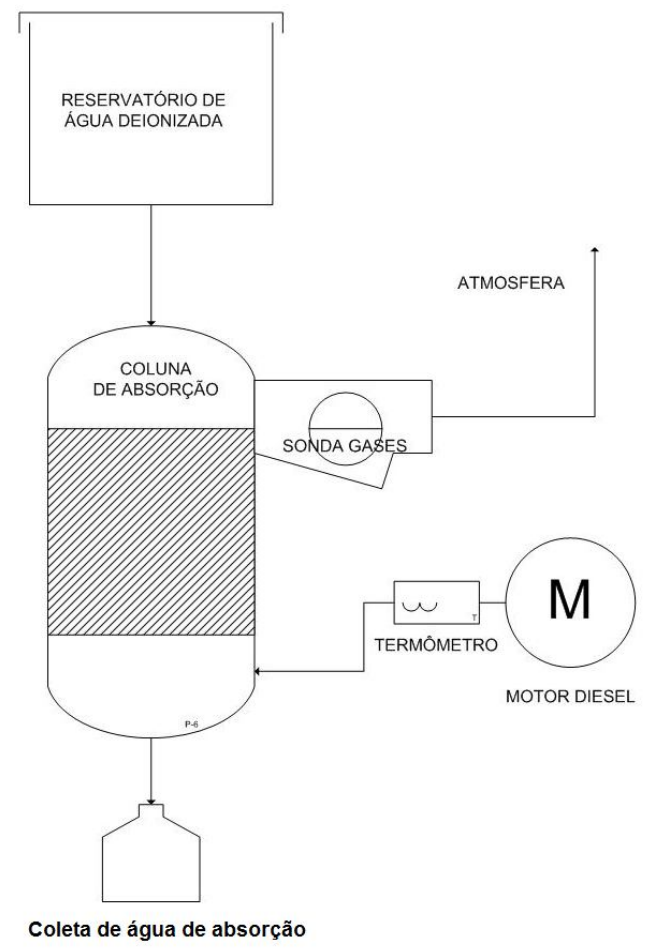

Figura 1 - Diagrama esquemático do sistema de absorção de gases.

Fonte: Do autor, 2014.

Os experimentos foram realizados em triplicata, com o processo de combustão do diesel durante um período de 15 minutos, sendo estabelecido como tempo zero o início de partida do motor a frio. Foram estabelecidos intervalos de tempo de 1 minuto para a leitura de NOx e $\mathrm{CO}$, e o equipamento utilizado para essa mensuração foi o analisador de gases de combustão Confor Optima 7 (Figura 2a). A quantificação de HPA's foi realizada após 15 minutos de experimento com o auxílio da sonda HPA Enviroflu da marca TRIOS (Figura 2b). 

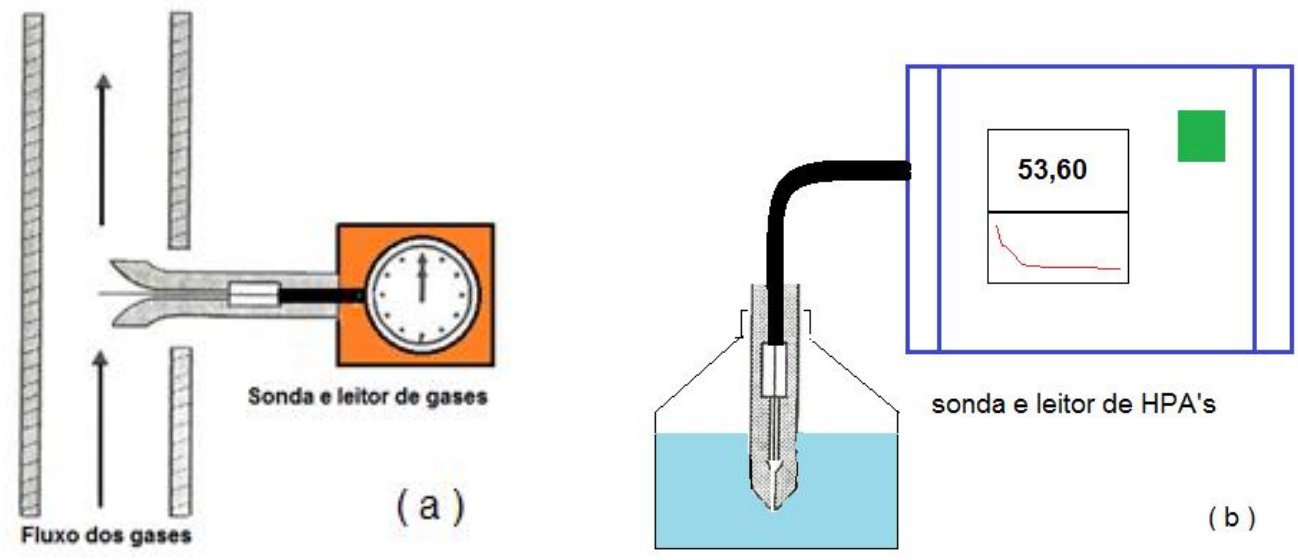

Figura 2 - Equipamento utilizado para: (a) quantificação de gases da combustão; (b) quantificação de HPA's.

Fonte: Do autor, 2014.

\section{RESULTADOS E DISCUSSÃO}

Os testes efetuados com a queima de diesel S10 e diesel S50 apresentaram valores de emissão similares tanto para o CO quanto para NOx, porém com pequena redução do diesel S-10 em relação do S-50. De acordo com a resolução CONAMA N N $^{\circ}$ 382/2006, a produção de óxidos nitrosos (NOx), refere-se à soma das concentrações de monóxido de nitrogênio (NO) e dióxido de nitrogênio $\left(\mathrm{NO}_{2}\right)$. A Figura 3 apresenta uma comparação das emissões de NOx provenientes do diesel S-10 e diesel S-50.

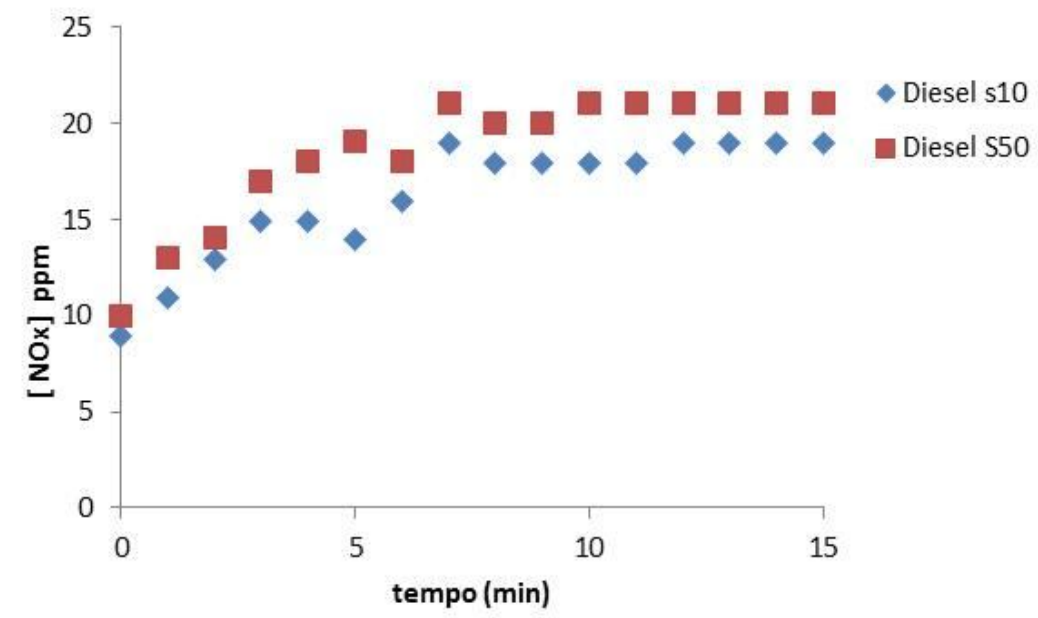

Figura 1 - Quantificação da emissão de NOx (ppm) do diesel s10 e diesel S-50. 
Conforme apresentado na Figura 3, a média da emissão de NOx do diesel S10 $(17,88$ ppm) nos ensaios realizados foi inferior a emissão do diesel S-50 (20,52 ppm). A redução na emissão de NOx proveniente do diesel S10 é justificada pelo elevado índice de cetanos (NC), diretamente relacionado com uma melhora na queima de combustível, que se traduz em mais potência e torque, e menor emissão de fumaça e óxidos de nitrogênio (NOx).

Os ensaios realizados para a combustão do diesel S10 (Tabela 1) e diesel S50 (Tabela 2) durante o intervalo de tempo de 15 minutos são apresentados a seguir.

Tabela 1 - Resultado médio relativo à medição de gases da queima do diesel S10.

\begin{tabular}{|c|c|c|c|c|c|c|c|}
\hline $\begin{array}{c}\text { tempo } \\
(\mathrm{min})\end{array}$ & $\% \mathrm{O}_{2}$ & $\begin{array}{c}\% \\
\mathrm{CO}_{2}\end{array}$ & $\begin{array}{l}\mathrm{ppm}^{*} \\
\mathrm{CO}\end{array}$ & $\begin{array}{c}\text { ppm } \\
\text { NO }\end{array}$ & $\begin{array}{l}\mathrm{ppm} \\
\mathrm{NO}_{2}\end{array}$ & $\begin{array}{l}\text { ppm } \\
\text { NOx }\end{array}$ & $\begin{array}{c}\text { Temperatura } \\
\left({ }^{\circ} \mathbf{C}\right)\end{array}$ \\
\hline 0 & 20,8 & 0,03 & 1590 & 5 & 7 & 9 & 35 \\
\hline 1 & 20,8 & 0,25 & 2475 & 6 & 4 & 10 & 154 \\
\hline 2 & 20,8 & 1,49 & 2365 & 9 & 3 & 12 & 185 \\
\hline 3 & 18,1 & 2,12 & 2893 & 11 & 3 & 14 & 190 \\
\hline 4 & 16,7 & 2,42 & 2458 & 12 & 2 & 14 & 186 \\
\hline 5 & 16,9 & 2,43 & 2026 & 14 & 2 & 15 & 170 \\
\hline 6 & 17,5 & 2,20 & 1861 & 13 & 2 & 15 & 178 \\
\hline 7 & 17,5 & 2,16 & 1843 & 14 & 3 & 17 & 176 \\
\hline 8 & 16,9 & 2,33 & 2016 & 15 & 3 & 18 & 166 \\
\hline 9 & 17,0 & 2,44 & 2193 & 14 & 4 & 18 & 172 \\
\hline 10 & 17,2 & 2,39 & 2435 & 14 & 4 & 18 & 168 \\
\hline 11 & 17,1 & 2,31 & 2484 & 14 & 4 & 18 & 165 \\
\hline 12 & 17,1 & 2,30 & 2513 & 15 & 4 & 19 & 157 \\
\hline 13 & 17,2 & 2,31 & 2522 & 15 & 4 & 19 & 168 \\
\hline 14 & 17,1 & 2,31 & 2593 & 15 & 4 & 19 & 168 \\
\hline 15 & 16,9 & 2,44 & 2558 & 15 & 4 & 19 & 168 \\
\hline
\end{tabular}

*ppm= partes por milhão. 
Tabela 2 - Resultado médio relativo à medição de gases da queima do diesel S50.

\begin{tabular}{|c|c|c|c|c|c|c|c|}
\hline $\begin{array}{c}\text { tempo } \\
(\mathbf{m i n})\end{array}$ & $\begin{array}{c}\boldsymbol{\%} \\
\mathbf{O}_{\mathbf{2}}\end{array}$ & $\begin{array}{c}\mathbf{\mathbf { C O } _ { 2 }} \\
\mathbf{\mathbf { P O }}\end{array}$ & $\begin{array}{c}\mathbf{\text { ppm }} \\
\mathbf{N O}\end{array}$ & $\begin{array}{c}\mathbf{p p m} \\
\mathbf{N O}_{\mathbf{2}}\end{array}$ & $\begin{array}{c}\mathbf{p p m} \\
\mathbf{N O x}\end{array}$ & $\begin{array}{c}\text { Temperatura } \\
\left({ }^{\circ} \mathbf{C}\right)\end{array}$ \\
\hline 0 & 17,4 & 0,02 & 1316 & 7 & 3 & 10 & 35 \\
\hline 1 & 17,0 & 2,28 & 2369 & 10 & 3 & 13 & 182 \\
\hline 2 & 17,0 & 2,28 & 2500 & 11 & 3 & 14 & 234 \\
\hline 3 & 16,8 & 2,43 & 2563 & 14 & 3 & 17 & 252 \\
\hline 4 & 16,7 & 2,50 & 2710 & 16 & 2 & 18 & 249 \\
\hline 5 & 16,9 & 2,43 & 2761 & 17 & 2 & 19 & 241 \\
\hline 6 & 18,7 & 2,40 & 2613 & 16 & 2 & 18 & 237 \\
\hline 7 & 16,6 & 2,38 & 1840 & 19 & 2 & 21 & 233 \\
\hline 8 & 16,5 & 2,56 & 2788 & 19 & 1 & 20 & 228 \\
\hline 9 & 16,6 & 2,59 & 2900 & 20 & 1 & 20 & 217 \\
\hline 10 & 16,6 & 2,60 & 2947 & 20 & 1 & 21 & 216 \\
\hline 11 & 16,6 & 2,58 & 2858 & 20 & 1 & 21 & 212 \\
\hline 12 & 16,6 & 2,57 & 2938 & 21 & 0 & 21 & 216 \\
\hline 13 & 16,6 & 2,53 & 2949 & 21 & 0 & 21 & 211 \\
\hline 14 & 16,6 & 2,54 & 2844 & 21 & 0 & 21 & 211 \\
\hline 15 & 16,7 & 2,48 & 2825 & 21 & 0 & 21 & 212 \\
\hline
\end{tabular}

*ppm= partes por milhão.

Os dados apresentados nas Tabelas 1 e 2, denota uma relação entre o aumento da temperatura dos gases de combustão e emissão de NO, e como consequência de NOx. A redução da emissão de NOx do diesel S10, mesmo sendo pouco significativa, traduz em uma vantagem principalmente pelos problemas ocasionados pela emissão de óxidos nitrosos na atmosfera, como o smog fotoquímico e a depleção do ozônio. O ensaio comprova que o nível de $\mathrm{NO}$ nos gases de exaustão é mais elevado que a de $\mathrm{NO}_{2}$ conforme reportado na literatura (Song, 2007). Desta forma a melhor forma de controle da emissão de NO e consequente emissão de NOx é o emprego de catalisador apropriado no sistema de escapamento dos gases de exaustão do motor.

A emissão de monóxido de carbono (CO) no motor estacionário foi acompanhada por meio do uso do multianalisador de gases Confor Optima 7. As concentrações foram determinadas na unidade ppm e convertidas em média de leitura na unidade $\mathrm{mg} / \mathrm{N} . \mathrm{m}^{3} \mathrm{de}$ ar de forma a comparar com o valor referencial da Resolução do Conama 382/2006 que estabelece 
os limites máximos de emissão de poluentes atmosféricos para fontes fixas. Os dados são apresentados na tabela 4 .

Tabela 4 - Resultado médio relativo à emissão de CO do Diesel S-10 e Diesel S-50.

\begin{tabular}{|c|c|c|c|}
\hline Parâmetro & Diesel S10 & Diesel S50 & Conama 382/2006 \\
\hline $\mathrm{CO}\left(\mathrm{mg} / \mathrm{N} . \mathrm{m}^{3}\right)$ & 1660,39 & 1833 & 1830 \\
\hline $\begin{array}{c}\text { Temperatura média } \\
\text { de combustão }\end{array}$ & $200^{\circ} \mathrm{C}$ & $212^{\circ} \mathrm{C}$ & $----\mathrm{X}----$ \\
\hline
\end{tabular}

Conforme apresentado na Tabela 4, as emissões de monóxido de carbono (CO) tanto para o Diesel S10 quanto para o diesel S50 atendem a resolução do Conama 382/2006. Entretanto deve-se ressaltar que as condições da Resolução são estabelecidas através das Condições Normais de temperatura e Pressão (CNTP).

De acordo com os testes de HPA realizadas com o diesel S10 e S50, pode-se perceber que a concentração média final da concentração de HPA no diesel S10 (36 $\mu \mathrm{g} / \mathrm{L})$ é menor quando comparados ao diesel S50 $(53 \mu \mathrm{g} / \mathrm{L})$. Os resultados dos testes efetuados foram avaliados com as condições de operação do motor estacionário na queima de diesel S10 e diesel S50. Uma possível explicação se deve ao índice de cetano (NC) do diesel S50 ser inferior ao do diesel S10. A escala NC, conceitualmente similar à escala de octanagem utilizada para a gasolina foi estabelecida para descrever a qualidade de ignição do diesel e seus componentes. Neste caso, um NC mais alto (caso do diesel S10), permite uma relação de mistura combustível/ar mais eficiente para a combustão e temperaturas de queima mais baixas, conforme os dados apresentados e discutidos nos ensaios.

\section{CONCLUSÃO}

A partir dos resultados obtidos, foi possível observar que o diesel S10 apresenta uma pequena redução na emissão de poluentes comparativamente ao diesel S50. Os experimentos em triplicata demonstraram que a emissão de óxidos nitrosos (NOx) e de monóxido de carbono foram inferiores as emissões provenientes do diesel S-50. A emissão de CO no motor estacionário apresentou valor de $1660,39 \mathrm{mg} / \mathrm{N} \cdot \mathrm{m}^{3}, 9,26 \%$ inferior estabelecido pela resolução do Conama 382/2006 (1830 mg/N.m³). O diesel S10 apresentou valor de emissão de CO $\left(1833 \mathrm{mg} / \mathrm{N} . \mathrm{m}^{3}\right)$ no limite estabelecido pela mesma resolução. 


\section{REFERÊNCIAS}

BUNGER, J., Krahl, J., Franke, H.-U., Munack, A. and Hallier, E. Mutagenic and cytotoxic effects of exhaust particulate matter of biodiesel compared to fossil diesel fuel. (1998) Mutation Research/Genetic Toxicology and Environmental Mutagenesis 415 (1-2), 13-23, 1998.

BOUROTTE, C.; FORTI, M.-C.; TANIGUCHI, S.; BÍCEGO, M. C.; LOTUFO, P. A. A wintertime study of PAHs in fine and coarse aerosols in São Paulo city, Brazil. Atmospheric Environment. Issue 21, v.39, p.3799-3811, 2005.

BRASIL. Resolução CONAMA n 382, de 26 de dezembro de 2006: "Estabelece os limites máximos de emissão de poluentes atmosféricos para fontes fixas", 2006.

CORREA, S. and ARBILLA G." Aromatic hydrocarbons emissions in diesel and biodiesel exhaust. Atmospheric Environment 40, 6821-6826, 2006.

FYNLAYSON-PITTS, B.J.; pitts Jr, J.N. Chemestry of upper and lower atmosphere - Theory, experiments, and applications. New York: Academic, 969p., 2000.

KNOTHE G, Van Gerpen J, Krahl J (eds) The biodiesel handbook. AOCS Press, Champaign, IL, 2005.

KOZERSKI, G. R.; HESS, S. C. Estimativa dos poluentes emitidos pelos ônibus e microônibus de Campo Grande/MS, empregando como combustível diesel, biodiesel ou gás natural. Engenharia Sanitaria e Ambiental. v.11, p.113-117, 2006.

SCHRÖDER, O.; BÜNGER, J.; MUNACK, A.; KNOTHE, G.; KRAHL, J. Exhaust emissions and mutagenic effects of diesel fuel, biodiesel and biodiesel blends. Fuel. Issue 0, v.103, p.414420, 2013.

SONG, C.-L.; ZHOU, Y.-C.; HUANG, R.-J.; WANG, Y.-Q.; HUANG, Q.-F.; LÜ, G.; LIU, K.-M. Influence of ethanol-diesel blended fuels on diesel exhaust emissions and mutagenic and genotoxic activities of particulate extracts. Journal of Hazardous Materials. Issue 2, v.149, p.355363, 2007.

TOMASZEWSKI, J. E.; SMITHENRY, D. W.; CHO, Y.-M.; LUTHY, R. G.; LOWRY, G. V.; REIBLE, D.; MACEK, T.; SURÁ, M.; CHRASTILOVA, Z.; DEMNEROVA, K.; MACKOVÁ, M.; PAVLIKOVÁ, D.; SZEKERES, M.; SYLVESTRE, M. Treatment and Containment of Contaminated Sediments. In: D. Reible e T. Lanczos (Ed.). Assessment and Remediation of Contaminated Sediments: Springer Netherlands. Treatment and Containment of Contaminated Sediments, p.pp 137-178, 2006.

TSUKUE, N., OKUMURA, H., Ito, T., SUGIYAMA, G. and NAKAGIMA, T. Toxicological evaluation of diesel emissions on A549 cells. Toxicology in Vitro 24 (2), 363-369, 2010. 